\title{
Impact Des Facteurs Intersubjectifs Sur \\ L'engagement Des Cadres Ivoiriens Au Développement De Leur Localité d'Origine
}

\author{
Daboné Inoussa (Docteur) \\ Université Félix Houphouët-Boigny, \\ Département de Psychologie, Abidjan-Côte d'Ivoire
}

Méité Zoumana (Docteur)

Institut National de la Jeunesse et des Sports (INJS), Abidjan-Côte d'Ivoire

Doi:10.19044/esj.2018.v14n32p237 URL:http://dx.doi.org/10.19044/esj.2018.v14n32p237

\begin{abstract}
Despite the policy of developing localities initiated by the Ivorian state, disparities between rural and urban populations are still obvious. The lack of interest in this process of development from localities' native executives is highlighted. The present survey tempts to examine this lack of personal commitment from executives in relation with intersubjective factors such as sympathy, autonomy, mystical practices and communication. In so doing, a sample of 121 executives from the Ivorian public administration are selected rationally in the purpose of data collection through a questionnaire. Statistical analysis indicate that, except the autonomy to bring innovations in rural areas, the targeted intersubjective factors have a significant impact on the executives' level of commitment in their locality's development. As regards to practical repercussions, these results underline the necessity to develop the local populations' mind of sympathy and communication towards their executives. Local populations have also to put an end to any mystical practice able to frighten their native executives if they want them to fully take part in the development of their different localities.
\end{abstract}

Keywords: Ivory Coast, executives, commitment, intersubjective factors

\section{Résumé}

Malgré la politique de mise en valeur des localités initiée par l'État ivoirien, les inégalités entre les populations rurales et citadines demeurent criantes. Le désintérêt des cadres originaires des différentes localités à l'égard de ce processus se trouve notamment mis en cause. La présente étude tente alors d'examiner ce manque d'engagement personnel des cadres en relation avec les facteurs intersubjectifs tels que la sympathie, l'autonomie, les 
pratiques mystiques et la communication. À cet effet, 121 cadres de l'administration publique ivoirienne sont sélectionnés par choix raisonné en vue de la collecte de données par voie de questionnaire. Les traitements statistiques effectués ont permis d'aboutir aux résultats selon lesquels, à l'exception de l'autonomie d'apporter des innovations au milieu local, les facteurs intersubjectifs ciblés ont un impact significatif sur le niveau d'engagement des cadres au développement de leur localité. Sur le plan des retombées pratiques, ces résultats soulignent la nécessité de développer l'esprit de sympathie et de communication des populations locales vis-à-vis de leurs cadres et de lutter contre toute pratique mystique susceptible de les effrayer, de sorte à leur permettre d'apporter leur nécessaire contribution au développement des différentes localités du pays.

Mots-clés: Côte d'Ivoire, cadres, engagement, facteurs intersubjectifs

\section{INTRODUCTION ET PROBLEMATIQUE}

La Côte d'Ivoire connait ces dernières années une nette croissance dans la plupart des branches d'activités. Selon l'INS (2018), l'activité économique du pays en 2017 s'est accrue de 7,6\% (taux de croissance réel du PIB) par rapport à 2016. Ces performances globales n'ont cependant eu qu'un impact limité sur le taux de pauvreté encore estimé à $47 \%$ avec une grande disparité entre les revenus des citadins et ceux des populations rurales (APR, 2017). Cela fait dire à Faujas (2017) que l'économie ivoirienne se redresse mais la pauvreté s'accroît. Manciaux (2017) lui emboîte le pas en affirmant qu'il subsiste au sein du pays de fortes disparités aussi bien sur le plan du niveau d'instruction que sur le plan de l'emploi et du revenu. Etant donné que l'État ivoirien, conscient de ce que l'économie du pays repose sur l'agriculture, attache une importance particulière au développement des milieux ruraux, comment peut-on alors expliquer un tel résultat? Entre échec des politiques et déficit d'appropriation du processus de développement par les populations elles-mêmes, se situe la question épineuse de la participation des cadres au développement de leur localité d'origine.

Dans cette perspective, la présente étude s'intéresse aux facteurs qui pourraient rendre les activités de développement local plus attractives aux yeux des cadres. Ainsi prend-elle en compte, en vue d'une meilleure compréhension de la participation des cadres au développement de leur localité d'origine, la variable engagement organisationnel. Cette variable largement étudiée au niveau de l'entreprise s'impose dans le développement local. Le choix d'étudier l'engagement des cadres au développement local selon les facteurs intersubjectifs se justifie par le fait que les rapports entre le cadre et sa localité d'origine sont caractérisés par un ensemble de facteurs tels que la sympathie, l'autonomie, le climat social et la communication. L'étude de l'impact de ces 
facteurs intersubjectifs sur l'engagement des cadres au développement local présente alors un double intérêt scientifique et social.

D'un point de vue scientifique, les études relatives au développement local se caractérisent par leur complémentarité dans la mesure où elles mettent l'accent soit sur la dimension technique, soit sur la dimension politique, soit sur la dimension utilitaire de la participation des acteurs (Chauveau \& Lavigne Delville, 2013 ; Bonnal, 1997). La dimension technique renvoie au moyen d'amener les paysans à exprimer leurs besoins de développement. Elle a cependant l'inconvénient d'être animée par des acteurs extérieurs. $L a$ dimension politique cherche à réduire les inégalités à la base des mécanismes d'exclusion. Elle constitue le principe de base de la politique de décentralisation. La dimension utilitaire se présente comme un moyen d'assurer l'appropriation et l'adhésion des populations aux projets dans le cadre des stratégies actuelles de lutte contre la pauvreté. Á ce niveau, l'on souligne le rôle des organisations de la société civile pour encourager les populations à s'organiser, se concerter et s'exprimer afin que les politiques de développement prennent en compte leurs intérêts. Cette dimension utilitaire ne fait cependant guère mention de la participation des cadres au développement local. De plus, les environnements ruraux sont caractérisés par l'interaction permanente entre différentes composantes physiques, humaines sociales et culturelles. La présente étude essaie alors de comprendre comment le milieu d'origine peut jouer sur l'engagement des cadres au développement de leur localité d'origine. Elle permettra ainsi d'apporter une contribution à la connaissance des facteurs influençant ce phénomène.

Du point de vue social, cette étude a pour intérêt d'aider à résoudre le problème de la participation des cadres aux activités et initiatives de développement de leur localité d'origine. C'est en sachant précisément les facteurs qui entravent cet engagement que l'on pourra élaborer des plans cohérents d'incitation prenant en compte les besoins essentiels des cadres, leurs croyances et les éléments qu'ils valorisent afin d'assurer un meilleur développement des différentes localités. Cependant, les effets de l'engagement ne sont pas toujours directement observables et des confusions sont souvent relevées entre les termes. Le concept d'engagement est utilisé par différents intervenants dans divers domaines (Mowday, Porter \& Steers, 1982). À l'intérieur de l'organisation, Firestone \& Rosenblum (1988) distinguent trois catégories d'engagements. La première est l'engagement envers les membres de l'organisation. Elle vise à comprendre la façon dont on pourrait les motiver et les soutenir davantage. La deuxième est l'engagement envers l'activité. Elle vise à encourager le travailleur (le "cadre" dans notre étude) à l'utilisation de moyens visant à le rendre plus efficace. La troisième est l'engagement organisationnel. Pour ces auteurs, l'engagement organisationnel regroupe l'engagement envers l'organisation (l'esprit communautaire, l'identification et 
l'intégration) ainsi que ses objectifs (réfléchir constamment à la façon de renforcer la participation des populations et d'accroître leur performance. Particulièrement, les cadres originaires de ces localités devraient investir du temps dans la planification et dans la préparation des activités de développement local.

Pour définir l'engagement organisationnel, les auteurs se réfèrent classiquement à Porter et al. (1974) qui le conçoivent comme étant l'identification d'un individu à son organisation et son implication dans celleci. Les individus qui font preuve d'engagement sont caractérisés par un partage des valeurs de l'organisation, le désir de demeurer à son service et une disposition à donner des efforts au nom de celle-ci. Pourtant le lien avec l'organisation peut, en dehors de l'identification de l'individu aux valeurs de l'organisation, être fondé sur d'autres phénomènes formels ou informels. Á ce sujet, Meyer \& Allen (1997) relèvent deux grandes approches de l'engagement organisationnel : l'approche des points de focalisation (qui propose que les individus puissent être engagés envers plusieurs éléments à l'intérieur de l'organisation) et l'approche des fondements (qui suppose que l'engagement organisationnel puisse varier selon la nature de la relation qu'a l'individu avec l'organisation). Ainsi, un cadre peut être engagé envers son village pour des raisons affectives, matérielles ou morales. En plus de ces deux approches, Meyer \& Allen (1997) présentent l'engagement organisationnel dans une perspective multidimensionnelle. Celui-ci aurait ainsi trois dimensions: d'abord l'engagement affectif qui fait appel aux émotions, aux valeurs et se fait donc par désir et par choix; ensuite l'engagement de continuité qui réfère à la conscience que l'individu a des désavantages associés au fait d'abandonner son organisation; enfin l'engagement normatif qui se rapporte aux normes personnelles, morales et sociales : la personne s'engage par devoir ou par obligation. Par exemple, un cadre peut être engagé envers son village parce qu'il s'agit d'un "devoir". Selon toujours ces auteurs, les facteurs pouvant impacter l'engagement organisationnel sont de trois ordres: les caractéristiques personnelles (âge, sexe, scolarité, statut socio-économique, marital), la structure organisationnelle (utilisation du pouvoir de décision, clarté des processus décisionnels, climat d'apprentissage, degré de liberté dans la prise de décision) et les expériences de travail (soit contribuent au bien-être physique et psychologique, soit valorisent et mettent en évidence les compétences de l'individu au sein de l'organisation). Une interaction est évidemment possible entre ces facteurs et un cadre engagé représente une ressource importante pour le développement de sa localité d'origine.

Le développement local est défini par Uma (1977) comme étant l'accession à un meilleur niveau de vie de la grande masse des individus à faible revenu résidant dans les zones rurales et la possibilité pour eux de maintenir ce niveau de vie par leurs propres moyens. Dès lors, ce 
développement est un phénomène qualitatif qui ne se réfère pas exclusivement à l'expression quantitative de "croissance économique". Il résulte d'un processus cognitif qui modèle les attitudes des individus et qui leur permet de créer la richesse. Il ne suffit donc pas que les autorités souhaitent le développement, mais l'adhésion et l'apport des populations aux processus de développement s'avère indispensable. À ce niveau, l'engagement des cadres originaires de ces localités est nécessaire pour expliquer, faciliter, enrichir, soutenir et consolider les projets de développement entrepris. Le terme de cadre, dont la définition renvoie aux premiers travaux de Fayol (1918), désigne une catégorie d'individus dont le travail consiste à assurer des activités de direction, de coordination et de prise de décision. Ayant accédé à ce statut soit dès le premier emploi - du fait de leur diplôme - soit par promotion, les cadres apparaissent comme étant un potentiel humain important pour toute organisation étant donné leur rôle de tout premier ordre dans la conception, l'élaboration et la mise en œuvre des décisions affectant de façon décisive la production. À ce titre, leur engagement peut être déterminant dans la réalisation des objectifs de développement local.

En remontant dans l'histoire, l'on peut noter qu'avant les années 1980, l'État ivoirien faisait de la mise en valeur des localités son affaire exclusive. Cette initiative ne suscita chez les cadres locaux et urbains qu'une indifférence se traduisant par leur désintérêt et leur manque d'implication personnelle. Si des voix n'hésitèrent pas à imputer la persistance du déficit de développements locaux à l'État qu'ils accusent d'avoir failli à sa mission, d'autres doigts accusateurs pointent par contre les cadres de n'avoir rien fait pour empêcher ce déficit.

Entre l'explication d'une politique de décentralisation mal élaborée par l'État et celle d'une population locale - y compris ses cadres - n'ayant pas joué son rôle pour que le rêve de développement devienne une réalité, une évidence demeure: le développement ne peut se concevoir sans l'esprit de responsabilité, de créativité et d'initiative des populations elles-mêmes. Le projet de développement local a même été lié au processus de décentralisation (Chauveau \& Lavigne Delville, 2013) dans le but de créer les conditions d'une participation effective de toutes les forces, y compris les populations locales elles-mêmes. Il est dès lors indispensable que la catégorie des cadres, si habituée aux prises d'initiatives et de décisions, s'y engage dans la perspective de réalisation des indicateurs clés du développement. Ces indicateurs clés concernent selon Cameliau (1996) des domaines aussi variés que l'école, la santé, l'eau, l'électricité et l'amélioration des revenus. Le défi majeur est cependant d'amener les cadres à une participation effective au processus de développement local. Étant donné la puissance des aspects psychologiques individuels et collectifs - dans la réussite des plans de développement local (Iverson \& Buttigieig, 1999), une question majeure qui se pose alors est 
relative au rapport entre le cadre et les populations locales: les facteurs intersubjectifs ont-ils un impact sur l'engagement des cadres au développement local?

Une étude sur l'engagement des cadres ivoiriens au développement de leur localité, en rapport avec les facteurs intersubjectifs, s'avère alors intéressante dans la perspective de découverte des raisons profondes de l'échec des projets de développement local. Cette étude a pour objectif de distinguer ce qui, du point de vue des facteurs intersubjectifs, organise et sous-tend l'engagement des cadres au développement local. Plus précisément, il s'agit de savoir si l'engagement des cadres au développement local est tributaire des facteurs intersubjectifs tels que la sympathie, l'autonomie, le climat social et la communication dans les relations entre les cadres et les habitants de la localité d'origine.

Dans cette perspective, le cadre constitué par les théories de l'interdépendance sociale (Kelley \& Thibaut, 1978) et de l'engagement (Joule \& Beauvois, 1999) apporte un éclairage utile à la compréhension du phénomène d'engagement des cadres au développement local. La théorie de l'interdépendance sociale stipule que les partenaires manifestent, dans l'échange de ressources, une dépendance mutuelle dont les indicateurs sont la fréquence des influences réciproques, la diversité des domaines ainsi que la force (vitesse et amplitude) de ces influences mutuelles. La théorie de l'engagement stipule que lorsqu'un acte initial est réalisé par une personne, il constitue, suivant les traits de la situation, un acte préparatoire "engageant" insérant la personne dans un cours d'actions qui la conduiront à aller au-delà de l'acte initial. Le niveau d'engagement ainsi réalisé varie selon le nombre d'actes engageants, l'importance du comportement pour le sujet, le sentiment de liberté perçue par le sujet ainsi que le caractère irrévocable de l'engagement. Ces considérations nous permettent d'émettre les hypothèses suivantes: Hypothèse 1: le fait de bénéficier de la sympathie de la population de sa localité d'origine favorise l'engagement du cadre au développement local, Hypothèse 2: l'autonomie d'apporter des innovations dans sa localité d'origine favorise l'engagement du cadre au développement local, Hypothèse 3: Les pratiques mystiques locales défavorisent l'engagement du cadre au développement local, Hypothèse4: Le fait de se sentir écouté par la population locale favorise l'engagement du cadre au développement local. La vérification de ces hypothèses commande une investigation sur le terrain. Il s'avère alors nécessaire de préciser la méthodologie utilisée pour conduire cette investigation. 


\section{METHODOLOGIE}

\section{II.1. Population et échantillon}

L'étude a été menée auprès de 121 cadres abidjanais, dont 58 femmes et 63 hommes, sélectionnés par choix raisonné. Le choix d'Abidjan comme terrain d'étude répond à plusieurs critères. Principale ville du pays et siège de l'administration centrale, cette métropole a une forte concentration de hauts cadres issus de toutes les régions et repartis dans tous les secteurs de l'administration publique. Les répartitions des cadres sélectionnés sont comparables selon le sexe (valeur maximale du test $\chi 2=1$, ddl=1; ns) et selon les régions du pays, comme indiqué dans le tableau suivant.

Tableau 1: Répartition des enquêtés selon le sexe et la région d'origine

\begin{tabular}{|c|c|c|c|}
\hline Régions & Femmes & Hommes & Total \\
\hline Région de l'Agnéby-Tiassa & 1 & 1 & 2 \\
\hline Région du Bafing & 2 & 2 & 4 \\
\hline Région de la Bagoué & 1 & 2 & 3 \\
\hline Région du Bélier & 1 & 2 & 3 \\
\hline Région du Béré & 1 & 2 & 3 \\
\hline Région du Bounkani & 2 & 1 & 3 \\
\hline Région du Cavally & 2 & 2 & 4 \\
\hline Région du Folon & 2 & 2 & 4 \\
\hline Région de Gbêkê & 2 & 2 & 4 \\
\hline Région du Gbôklé & 3 & 1 & 4 \\
\hline Région du Gôh & 2 & 2 & 4 \\
\hline Région du Gontougo & 2 & 3 & 5 \\
\hline Région du Guémon & 3 & 2 & 5 \\
\hline Région du Hambol & 2 & 2 & 4 \\
\hline Région du Haut-Sassandra & 1 & 2 & 3 \\
\hline Région de l'Iffou & 2 & 3 & 5 \\
\hline Région de l'Indénié-Djuablin & 3 & 2 & 5 \\
\hline Région du Kabadougou & 2 & 2 & 4 \\
\hline Région des Grands Ponts & 2 & 2 & 4 \\
\hline Région du Lôh-Djiboua & 2 & 2 & 4 \\
\hline Région de la Marahoué & 2 & 2 & 4 \\
\hline Région de la Mé & 2 & 2 & 4 \\
\hline Région de la Nawa & 2 & 2 & 4 \\
\hline Région du N'zi & 2 & 3 & 5 \\
\hline Région du Poro & 2 & 2 & 4 \\
\hline Région de San Pedro & 2 & 3 & 5 \\
\hline Région du Sud-Comoé & 2 & 3 & 5 \\
\hline Région du Tchologo & 2 & 1 & 3 \\
\hline Région du Tonkpi & 2 & 3 & 5 \\
\hline Région du Worodougou & 2 & 3 & 5 \\
\hline Total & 58 & 63 & 121 \\
\hline Test statistique $\chi 2$ corr & $4,069 \mathrm{~ns}$ & $5,095 \mathrm{~ns}$ & $4,702 \mathrm{~ns}$ \\
\hline
\end{tabular}

Les régions contenues dans ce tableau sont au nombre de trente selon le Décret $\mathrm{n}^{\circ}$ 2011-263 du 28 septembre 2011 portant organisation du territoire national en Districts et en Régions. L'estimation de la significativité des écarts 
entre les effectifs des cadres par région d'origine montre que ces effectifs sont équivalents non seulement globalement $(\chi 2=4,702$, ddl $=29$; ns $)$ mais aussi chez les femmes $(\chi 2=4,069$, ddl $=29 ; n s)$ et chez les hommes interrogés $(\chi 2=5,095$, ddl=29; ns). Les cadres ainsi sélectionnés ont accepté de répondre à l'instrument d'étude.

\section{II.2. Instrument de mesure}

L'instrument utilisé pour la collecte des données est un questionnaire pluridimensionnel. La première dimension concerne les données biographiques portant sur le sexe et la région d'origine des cadres interrogés. La seconde dimension appréhende les quatre facteurs intersubjectifs sympathie, autonomie, pratiques mystiques et communication - tous de nature qualitative avec deux modalités "oui" ou "non". Ainsi, le facteur sympathie conduira le cadre à indiquer si les populations de sa localité d'origine sont sympathiques (aimables avec lui) ou non. Au niveau du facteur autonomie, le cadre interrogé indique s'il est autonome (agit avec liberté) ou non dans les innovations qu'il apporte dans sa localité d'origine. Pour le facteur "pratiques mystiques" il doit indiquer s'il y a des pratiques mystiques dans sa localité ou non. Au niveau du facteur communication, il indiquera s'il se sent écouté ou non par les populations de sa localité d'origine. La troisième dimension de l'instrument est constituée par la mesure de la variable dépendante "engagement dans le développement local". Celle-ci est de nature quantitative, car mesurée à l'aide d'une échelle de mesure en 5 points allant de "complètement en désaccord" à "complètement d'accord". Plus précisément, l'échelle d'engagement organisationnel de Meyer \& Allen traduite en français par Stoeva (2006) a été utilisée. Cette échelle comprend 18 items, soit respectivement 6 items pour chacune des composantes - affectif, continuité et normatif - de l'engagement organisationnel. L'échelle a été soumise à une procédure d'adaptation au milieu ivoirien. A cet effet, elle a été présentée à 10 cadres abidjanais invités à inscrire la lettre A devant tout item qu'ils trouvent ambigu. Une côte d'ambiguïté a alors été calculée pour chaque item dans la perspective d'une reformulation de tout item ayant une côte d'ambiguïté d'au moins $50 \%$. Le questionnaire ainsi obtenu a été soumis à 15 cadres de l'administration publique. Cette phase a visé à tester l'accueil et la présentation de l'instrument, de même que le temps de passation. Nous obtenons en définitive un questionnaire dont la passation dure de 15 à 22 minutes, avec une échelle d'engagement organisationnel dont le coefficient de fidélité alpha de Cronbach est de 0,91.

Chacun des cadres contactés a été interrogé sur le lieu du travail en fonction de sa disponibilité. Ceux-ci ont tous répondu à l'instrument en notre présence, ce qui a eu l'avantage d'obtenir des questionnaires correctement remplis par les personnes concernées. 
Les questionnaires ainsi remplis ont été dépouillés en vue de l'analyse. Concernant l'échelle d'engagement ce dépouillement a consisté à accorder une valeur numérique à chaque réponse suivant ce principe: 1 pour "Complètement en désaccord", 2 pour "en désaccord", 3 pour "ni d'accord ni en désaccord", 4 pour "d'accord" et 5 pour "complètement d'accord". La somme des points obtenus par un sujet correspond à la mesure de son engagement dans le développement de sa localité d'origine, variant dans les limites théoriques de 18 à 90 .

L'analyse des données relatives à chacun des facteurs intersubjectifs a été effectuée à l'aide du $t$ de Student pour échantillons indépendants, en nous assurant chaque fois de l'homogénéité des variances dans la perspective de l'analyse de leur impact sur l'engagement au développement local. L'analyse de variance est utilisée pour tester l'impact conjoint des facteurs intersubjectifs sur l'engagement au développement local. Toutes les analyses ont été effectuées à l'aide du programme SPSS.

\section{RÉSULTATS}

Au terme de l'analyse menée dans le cadre des différentes hypothèses émises, cinq principaux résultats émergent. Ces résultats sont relatifs à l'impact des facteurs intersubjectifs sur l'engagement du cadre ivoirien au développement de sa localité d'origine. Les indices d'asymétrie et d'aplatissement sont respectivement de $-0,216$ et $-0,953$, ce qui atteste de la normalité de la distribution des données relatives à l'engagement au développement local chez les cadres.

\section{III.1. Sympathie des populations et engagement des cadres au développement local}

Le groupe des cadres bénéficiant de la sympathie des populations locales et celui des cadres qui ne bénéficient pas d'une telle sympathie étant comparables d'après le test d'homogénéité des variances de Levene $(\mathrm{F}=0,960$; ns), nous sommes fondé à utiliser le test de Student. Le tableau ci-dessous indique les caractéristiques des deux groupes de cadres.

Tableau 2 : Statistiques de groupes de cadres selon la sympathie dont ils bénéficient dans leur localité d'origine

\begin{tabular}{ccccc}
\hline Engagement au développement & $\mathrm{N}$ & Moyenne & Ecart-type \\
\hline \multirow{2}{*}{ Sympathie } & Non & 90 & 45,6111 & 17,90593 \\
\cline { 2 - 5 } & Oui & 31 & 69,9677 & 16,05580
\end{tabular}

L'on note d'après ce tableau que la moyenne des cadres qui bénéficient de la sympathie des populations de leur localité $(\mathrm{m}=69,9677)$ est supérieure à celle des cadres qui n'en bénéficient pas $(\mathrm{m}=45,6111)$. La significativité de ces différences est fournie par le tableau suivant: 
Tableau 3 : Impact du facteur sympathie sur l'engagement au développement local Test-t pour égalité des moyennes

Engagement au développement

T ddl

Sig. (bilatérale)

\begin{tabular}{cccc}
\hline Hypothèse de variances égales & $-6,873$ & 119 & 0,000 \\
\hline Hypothèse de variances inégales & $-7,727$ & 65,965 & 0,000 \\
\hline
\end{tabular}

Selon ce tableau $\mathrm{t}=-6,873$ ( $119 \mathrm{ddl} ; \mathrm{p}<0.01)$. Cela confirme l'hypothèse H1 selon laquelle les cadres qui bénéficient de la sympathie des populations de leur localité sont plus engagés au développement local que ceux qui ne bénéficient pas d'une telle sympathie.

\section{III.2- Autonomie et engagement des cadres au développement local}

La condition de l'égalité des variances entre les groupes de cadres, selon que ceux-ci soient autonomes (libres de pouvoir apporter des innovations dans leur localité) ou non, nécessaire à l'utilisation du t de Student est vérifiée grâce au test de Levene $(\mathrm{F}=0,502 ; \mathrm{ns})$. Les deux groupes sont donc comparables et le tableau suivant nous instruit sur leurs caractéristiques.

Tableau 4: Statistiques des groupes de cadres selon leur autonomie d'apporter des innovations dans leur localité d'origine

\begin{tabular}{lcccc}
\hline \multicolumn{2}{c}{$\begin{array}{l}\text { Engagement au } \\
\text { développement }\end{array}$} & $\mathrm{N}$ & Moyenne & Ecart-type \\
\hline \multirow{2}{*}{ Autonomie } & Non & 53 & 52,7925 & 19,19872 \\
\cline { 2 - 5 } & Oui & 68 & 51,1176 & 20,76559 \\
\hline
\end{tabular}

Les résultats du tableau ci-dessus indiquent des moyennes d'engagement des deux groupes de cadres au développement local sensiblement similaires $(52,79$ et 51,11$)$. La significativité des écarts entre ces valeurs est fournie par le tableau ci-dessous.

Tableau 5: Impact du facteur autonomie sur l'engagement au développement local Test-t pour égalité des moyennes

\begin{tabular}{|c|c|c|c|c|c|c|c|}
\hline \multirow{3}{*}{$\begin{array}{l}\text { Engagement au } \\
\text { développement }\end{array}$} & \multicolumn{7}{|c|}{ Test-t pour égalité des moyennes } \\
\hline & \multirow[t]{2}{*}{$\mathrm{t}$} & \multirow[t]{2}{*}{ Ddl } & \multirow{2}{*}{$\begin{array}{l}\text { Sig. } \\
\text { (bilatéral } \\
\text { e) }\end{array}$} & \multirow{2}{*}{$\begin{array}{l}\text { Différenc } \\
\text { e } \\
\text { moyenne }\end{array}$} & \multirow{2}{*}{$\begin{array}{l}\text { Différence } \\
\text { écart-type }\end{array}$} & \multicolumn{2}{|c|}{$\begin{array}{c}\text { Intervalle de } \\
\text { confiance } 95 \% \text { de la } \\
\text { différence } \\
\end{array}$} \\
\hline & & & & & & $\begin{array}{c}\text { Inférieur } \\
\mathrm{e}\end{array}$ & Supérieure \\
\hline $\begin{array}{c}\text { Hypothèse de } \\
\text { variances égales }\end{array}$ & $\begin{array}{c}0,45 \\
5 \\
\end{array}$ & 119 & 0,650 & 1,67481 & 3,68221 & $-5,61634$ & 8,96595 \\
\hline $\begin{array}{c}\text { Hypothèse de } \\
\text { variances inégales }\end{array}$ & $\begin{array}{c}0,45 \\
9\end{array}$ & 115,52 & 0,647 & 1,67481 & 3,64635 & $-5,54756$ & 8,89718 \\
\hline
\end{tabular}

Selon ce tableau, $\mathrm{t}=0,455$ (119 ddl; ns). On peut donc conserver l'hypothèse nulle selon laquelle le niveau d'engagement des cadres au développement local ne dépend pas de leur autonomie d'apporter des innovations dans leur localité d'origine. 


\section{III.3. Pratiques mystiques et engagement au développement local}

Les variances des groupes de cadres, selon que des pratiques mystiques existent dans leur localité d'origine ou non, apparaissent comme étant similaires d'après le test de Levene $(\mathrm{F}=1,728 ; \mathrm{ns})$. Ces groupes sont donc comparables et leurs caractéristiques sont révélées par le tableau suivant.

Tableau 6: Statistiques de groupes de cadres selon qu'il y ait ou non des pratiques mystiques dans leur localité d'origine

\begin{tabular}{lcccc}
\hline \multicolumn{2}{c}{$\begin{array}{c}\text { Engagement au } \\
\text { développement }\end{array}$} & $\mathrm{N}$ & Moyenne & Ecart-type \\
\hline $\begin{array}{l}\text { Pratiques } \\
\text { mystiques }\end{array}$ & Non & 66 & 43,0303 & 17,62513 \\
\cline { 2 - 5 } & Oui & 55 & 62,4364 & 17,56023 \\
\hline
\end{tabular}

Ce tableau montre des moyennes d'engagement au développement différentes entre les deux groupes. Ces moyennes sont de 43,0303 et 62,4364. Le tableau suivant situe sur la significativité de leurs différences.

Tableau 7: Impact du facteur "pratiques mystiques" sur l'engagement au développement local

\begin{tabular}{cccc}
\hline & \multicolumn{3}{c}{ Test-t pour égalité des moyennes } \\
\cline { 2 - 4 } $\begin{array}{c}\text { Engagement au } \\
\text { développement }\end{array}$ & $\mathrm{t}$ & $\mathrm{Ddl}$ & Sig. (bilatérale) \\
\hline $\begin{array}{c}\text { Hypothèse de variances } \\
\text { égales }\end{array}$ & $-6,041$ & 119 & 0,000 \\
\hline $\begin{array}{c}\text { Hypothèse de variances } \\
\text { inégales }\end{array}$ & $-6,043$ & 115,247 & 0,000 \\
\hline
\end{tabular}

Il ressort de ce tableau que $\mathrm{t}=-6,041(119 \mathrm{ddl} ; \mathrm{p}<0,01)$. Cela permet de rejeter l'hypothèse nulle et de conserver l'hypothèse $\mathrm{H} 3$ selon laquelle les pratiques mystiques des populations locales ont un impact négatif sur l'engagement des cadres au développement de leur localité d'origine.

\section{III.4. Communication et engagement au développement local}

Les deux groupes de cadres, selon qu'ils se sentent écoutés dans leur localité d'origine ou non, apparaissent comme étant comparables dans la mesure où leurs variances sont similaires d'après le test de Levene $(\mathrm{F}=0,980$; ns). Cela nous fonde à utiliser le test de Student pour comparer le niveau d'engagement au développement local dans ces groupes de cadres. Le tableau suivant indique les caractéristiques de ces groupes.

Tableau 8: Statistiques de groupes de cadres selon qu'ils se sentent écoutés ou non dans leur localité d'origine

\begin{tabular}{ccccc}
\hline \multicolumn{2}{c}{$\begin{array}{c}\text { Engagement au } \\
\text { développement }\end{array}$} & $\mathrm{N}$ & Moyenne & Ecart-type \\
\hline \multirow{2}{*}{ Communication } & Non & 54 & 43,3889 & 18,74196 \\
\cline { 2 - 5 } & Oui & 67 & 58,6716 & 18,48045 \\
\hline
\end{tabular}


Ce tableau permet d'observer une différence entre les moyennes d'engagement au développement local sous les modalités de la variable communication. Plus précisément, les moyennes d'engagement au développement local sont plus élevées chez les cadres qui se sentent écoutés par les populations de leur localité d'origine $(\mathrm{m}=58,6716)$ que chez ceux qui ne se sentent pas écoutés par les populations $(\mathrm{m}=43,3889)$. La significativité de ces différences est fournie par le tableau suivant:

Tableau 9: Impact du facteur communication sur l'engagement au développement

\begin{tabular}{cccccc} 
& \multicolumn{5}{c}{ Tocal } \\
\cline { 2 - 6 } $\begin{array}{c}\text { Engagement au } \\
\text { développement }\end{array}$ & $\mathrm{t}$ & Ddl & $\begin{array}{c}\text { Sig. } \\
\text { (bilatérale) }\end{array}$ & $\begin{array}{c}\text { Différence } \\
\text { moyenne }\end{array}$ & $\begin{array}{c}\text { Différence } \\
\text { écart-type }\end{array}$ \\
\hline $\begin{array}{c}\text { Hypothèse de } \\
\text { variances égales }\end{array}$ & $-4,494$ & 119 & 0,000 & $-15,28275$ & 3,40103 \\
\hline
\end{tabular}

D'après ce tableau, $\mathrm{t}=-4,494 \quad(119 \mathrm{ddl} ; \mathrm{p}<0.01)$. Cela confirme l'hypothèse $\mathrm{H} 4$ selon laquelle les cadres qui se sentent écoutés par les populations locales sont plus engagés au développement de leur localité d'origine que ceux qui ne se sentent pas écoutés par les populations locales.

\section{III.5- Effets conjoints des facteurs intersubjectifs sur l'engagement au développement local}

Les résultats des interactions entre les quatre facteurs intersubjectifs et l'engagement des cadres au développement local sont synthétisés dans le tableau ci-dessous. On y trouve notamment les valeurs des $\mathrm{F}$ et leur significativité permettant de répondre à notre question de savoir si les variables sympathie, autonomie, pratiques mystiques et communication ont un impact sur l'engagement des cadres au développement local.

Tableau 10: Statistiques de l'effet d'interaction entre les facteurs intersubjectifs et l'engagement au développement local

Variable dépendante : Engagement au développement

\begin{tabular}{cccc}
\hline Source & ddl & F & Sig. \\
\hline Sympathie & 1 & 29,699 & 0,000 \\
Autonomie & 1 & 0,172 & 0,680 \\
Pratiques mystiques & 1 & 18,600 & 0,000 \\
Communication & 1 & 6,663 & 0,011 \\
Autonomie * Sympathie & 1 & 1,318 & 0,253 \\
Autonomie * Pratiques mystiques & 1 & 0,651 & 0,422 \\
Autonomie * Communication & 1 & 0,074 & 0,786 \\
Sympathie * Pratiques mystiques & 1 & 0,054 & 0,816 \\
Sympathie * Communication & 1 & 0,001 & 0,981 \\
Pratiques mystiques * Communication & 1 & 2,740 & 0,101 \\
Autonomie * Sympathie * Pratiques mystiques & 1 & 1,434 & 0,234 \\
Autonomie * Sympathie * Communication & 1 & 0,083 & 0,774 \\
Autonomie * Pratiques mystiques * Communication & 1 & 0,301 & 0,585 \\
Sympathie * Pratiques mystiques * Communication & 1 & 0,545 & 0,462 \\
Autonomie * Sympathie * Pratiques mystiques * Communicatio & 1 & 2,710 & 0,103 \\
\hline
\end{tabular}


Ce tableau confirme les effets spécifiques des facteurs intersubjectifs, mais aucune des combinaisons effectuées entre ces quatre facteurs n'indique d'effets conjoints significatifs sur l'engagement des cadres au développement local. Nous conservons donc l'hypothèse nulle selon laquelle les facteurs intersubjectifs n'ont pas d'effet interactif significatif sur le niveau d'engagement au développement local.

\section{DISCUSSION}

Sous l'objectif de savoir si l'engagement des cadres au développement de leur localité est conditionné par les facteurs intersubjectifs, cette étude abouti à des résultats indiquant que les facteurs sympathie, pratiques mystiques et communication ont un impact significatif sur l'engagement des cadres au développement local, par contre l'autonomie d'apporter des innovations au milieu local n'a pas d'impact sur ce phénomène

À la lumière des théories de l'interdépendance sociale (Kelley \& Thibaut, 1978) et de l'engagement (Joule \& Beauvois, 1999), l'on peut affirmer que les cadres manifestent vis-à-vis de leur localité d'origine une dépendance dont l'intensité est déterminée par la fréquence et la diversité de leurs échanges avec ces localités. Les comportements qu'ils manifestent dans la perspective de ces échanges constituent des "actes engageants" qui les amènent toujours à aller au-delà des engagements précédents. L'on comprend dès lors que les facteurs sympathie des populations locales, absences de pratiques mystiques et communication (se sentir écouté par les populations locales) constituent des facteurs qui poussent les cadres à augmenter la fréquence de leurs échanges avec leur localité d'origine, multipliant ainsi le nombre des actes engageants vis-à-vis de cette localité. Il n'est donc pas étonnant d'observer l'impact significatif de ces facteurs intersubjectifs sur l'engagement des cadres au développement de leur localité.

Boltanski (1993) indique que la sympathie est une capacité imaginative par laquelle nous pouvons nous sentir solidaires de personnes qui partagent d'autres conditions de vie que la nôtre. Selon lui la sympathie rend l'individu capable d'imaginer ce que l'autre ressent et d'en avoir de la compassion. Il rapproche ce concept de celui de l'altruisme qu'Organ (1988) inscrit parmi les dimensions associées de près à l'efficacité organisationnelle.

Nicourd (2008) met en lumière les processus de construction de l'engagement. Pour lui, ce phénomène porte l'empreinte du milieu d'origine (de naissance et de développement du cadre). Il explique qu'un des premiers facteurs favorisant l'engagement est l'environnement dans lequel l'individu a grandi: le fait d'avoir été membre d'une organisation de jeunesse ou d'une autre association dans son adolescence contribue à une poursuite de l'engagement à l'âge adulte. Cet engagement spécifique dans des mouvements de jeunesse se transmet ainsi au sein des familles à travers le processus de 
communication entre l'individu et ses proches. Si la disposition à l'engagement organisationnel peut être considérée comme potentiellement présente chez tous les enfants, son actualisation dépendra des échanges affectifs et cognitifs entre l'individu et les autres membres de cette organisation. S'il est confronté à des pratiques de justice, d'empathie, de solidarité etc., le niveau de son engagement sera élevé. Les facteurs intersubjectifs ciblés dans la présente étude - la sympathie des populations locales, les pratiques mystiques (sorcellerie) auxquelles elles ont recours, le sentiment d'être écouté par ces populations locales - entrent dans la perspective de ces pratiques décrites par Nicourd (2008) allant dans le sens d'un accroissement du niveau d'engagement des cadres au développement local. Ces explications trouvent un écho favorable chez Damon (1999) pour qui c'est progressivement que l'individu acquiert une identité morale à travers des milliers de petits événements tels que les commentaires des autres membres de l'organisation, l'observation de comportements qui l'inspirent ou le dégoûtent, la réflexion sur sa propre expérience, les influences familiales, scolaires ou des médias

Autour des actions de développement, deux univers de significations entrent en contact. Il y a d'une part le monde visible - celui du concret, du démontrable - et d'autre part le monde des invisibles comprenant des puissances (fétiches) et des esprits (y compris ceux des ancêtres). Selon Sanogo \& Coulibaly (2003), les cadres ont appris à analyser et à résoudre les situations et les phénomènes en faisant constamment recours aux recettes scientifiques et aux techniques modernes. Le fait que les populations rurales adoptent des croyances liées à l'invisible et l'indémontrable est à la base de plusieurs malentendus, voire des contradictions, autour des activités de développement des localités d'origine. Les invisibles seraient très puissants, se manifesteraient sous des formes diverses et se trouveraient partout parmi les vivants, dans la forêt, dans les cours d'eaux, sur les montagnes, etc. Ils auraient la capacité non seulement de veiller au bien-être des vivants, mais aussi de leur poser des problèmes quand ils sont fâchés. Pour les cadres, ces pratiques mystiques auxquelles les populations locales ont recours sont encore plus redoutables lorsqu'elles mettent en permanence en jeu la vie des individus. Dès lors, le recours à la sorcellerie pour le règlement des mécontentements de ces populations est vécu par les cadres comme une terreur incontrôlable de nature à les éloigner de leur localité d'origine.

\section{CONCLUSION}

L'engagement des cadres au développement local apparait comme étant un phénomène complexe qui demande la prise en compte de multiples facteurs dont ceux liés aux ressources humaines. Les facteurs psychologiques doivent être considérés et particulièrement les facteurs intersubjectifs qui sont des traits permettant de saisir la relation entre les cadres et leur population 
d'origine. Sous l'objectif d'étudier l'engagement des cadres au développement local en fonction des facteurs intersubjectifs telles que la sympathie, l'autonomie, les pratiques mystiques et la communication, cette recherche a été menée auprès de 121 cadres abidjanais des deux sexes et originaires des différentes régions du pays. Il ressort des résultats qu'à l'exception de l'autonomie, les facteurs intersubjectifs ciblés ont un impact significatif sur l'engagement des cadres au développement local. D'un point de vue pratique, l'enseignement principal qu'on en retire est que les cadres ont besoin d'un climat convivial et saint pour pouvoir donner le meilleur d'eux-mêmes au profit du développement des différentes localités du pays. Ces résultats montrent la nécessité de la sympathie et de l'esprit de communication au sein des populations rurales puis d'œuvrer à freiner toute pratique mystique susceptible d'apeurer les cadres. C'est ainsi que ces derniers se sentiront plus proches de leur localité d'origine et pourront être amenés à y apporter leur contribution nécessaire au développement. Le résultat concernant l'autonomie commande des études ultérieures en vue d'élucider davantage cette conception selon laquelle les cadres acceptent le fait que l'autonomie dans les actions ne soit pas concevable dans le contexte communautaire traditionnel.

\section{References:}

1. APR, Agence de Presse Régionale (2017). Côte d'Ivoire: Le taux de pauvreté passe à 47\%, [En ligne]: https://apr-news.fr/fr/actualites/cotedivoire-le-taux-de-pauvrete-passe-47, consulté le 22 août 2018.

2. Boltanski, L. (1993). La Souffrance à distance: Morale humanitaire, médias et politique, Paris: Métailié.

3. Bonnal, J. (1997). RED-IFO: an Analytical Model. Rural Development

4. Division. FAO, Rome. [En ligne]:

5. http://www.ciesin.org/decentralization/Frencwh/Tools/REDIFO.html;

6. consulté le 17 juillet 2018 .

7. Cameliau, C. (2016). L'économie à la recherche du développement: Crise d'une théorie, violence d'une pratique, Genève: Graduate Institute Publications.

8. Chauveau, J.P. \& Lavigne Delville, P. (2013). Développement Participatif, In I. Casillo, R. Barbier, L. Blondiaux, F. Chateauraynaud, J-M. Fourniau, R. Lefebvre, C. Neveu et D. Salles (Eds.), Dictionnaire critique et interdisciplinaire de la participation, Paris: GIS Démocratie et Participation.

9. Damon, W. (1999). Le développement moral des enfants, Pour la science, 264, 64-7.

10. Faujas, A. (2017). Côte d'Ivoire: l'économie se redresse mais la pauvreté s'accrôt, [En ligne]: https://www.jeuneafrique.com/mag/386937/economie/cote-divoire- 
leconomie-se-redresse-pauvrete-saccroit/, consulté le 13 septembre 2018.

11. Fayol, H. (1918). Administration industrielle et générale, Paris : Dunod.

12. INS, Institut National de la Statistique (2018). Comptes nationaux trimestriels 2018, une croissance continue, $\mathrm{n}^{\circ} 3$, Publication du premier trimestre 2018 des comptes nationaux trimestriels, INS Côte d'Ivoire, Direction de la Comptabilité Nationale, [En ligne]: http://www.ins.ci/n/documents/cnt/publication3.pdf, consulté le 22 août 2018.

13. Iverson, R. D. \& Buttigieig, D. M. (1999). Affective, normative and continuance commitment: can the "right kind" of commitment be managed?, Journal of Management Studies, 36(3), 307-333.

14. Joule, R.V. \& Beauvois, J.L. (1999). La soumission librement consentie, Paris : PUF

15. Kelley, H. H. \& Thibaut, J.W. (1978). Interpersonal Relations: A Theory of Interdependence, New York: John Wiley \& Sons.

16. Manciaux, C. (2017). Côte d'Ivoire : le FMI aussi attend plus de développement social, [En ligne]:

17. https://www.jeuneafrique.com/mag/386956/economie/cote-divoirefmi-attend-plus-de-developpement-social/; consulté le 13 septembre 2018

18. .Meyer, J. P. \& Allen, N. J. (1997). Commitment in workplace: theory, research and application, Tousand Oaks, London: Sage.

19. Mowday, R. T., Porter, L. W. \& Steers, R. M. (1982). Employeeorganisation linkages: The psychology of commitment, absenteeism and turnover, New York: Academic Press.

20. Nicourd, S. (2008). Qui s'engage aujourd'hui, Regards sociologiques sur la participation. Informations sociales, 1(145), 102-111

21. Organ, D.W. (1988). Organizational citizenship behavior: The good soldier syndrome, Lanham, MD : Lexington Books.

22. Porter, L. W., Steers, R M., Mowday, R M. \& Boulian, B. V. (1974). Organizational commitment, job satisfaction and turnover among psychiatry technicians, Journal of Applied Psychology, 59, 603-609

23. Sanogo, Z. \& Coulibaly, N. (2003). Croyances animistes et développement en Afrique subsaharienne. Horizons philosophiques, 13(2), 139-152.

24. Uma, L. (1976). Designing rural development programs: the lessons of past experience in Africa, Economic development and cultural change, 24, 289-308 